\title{
Magnetic microstructure of candidates for epitaxial dual Heusler magnetic tunnel junctions
}

\author{
A. Kaiser ${ }^{\text {a }}$, D. Banerjee ${ }^{\mathrm{a}}$, A.D. Rata ${ }^{\mathrm{b}}$, C. Wiemann ${ }^{\mathrm{a}}$, S. Cramm ${ }^{\mathrm{a}}$, and C.M. Schneider ${ }^{\mathrm{a}}$ \\ ${ }^{a}$ Forschungszentrum Jülich, Institut für Festkörperforschung IFF-9 and JARA-FIT, 52425 Jülich, Germany \\ ${ }^{\mathrm{b}}$ Leibniz-Institut für Festkörper- und Werkstoffforschung, Dresden, Germany
}

\begin{abstract}
Heusler alloys are considered as interesting ferromagnetic electrode materials for magnetic tunnel junctions, because of their high spin polarization. We therefore investigated the micromagnetic properties in a prototypical thin film system comprising two different Heusler phases $\mathrm{Co}_{2} \mathrm{MnSi}(\mathrm{CMS})$ and $\mathrm{Co}_{2} \mathrm{FeSi}$ (CFS) separated by a $\mathrm{MgO}$ barrier. The magnetic microstructure was investigated by x-ray photoemission microscopy (XPEEM). We find a strong influence of the Heusler phase formation process on the magnetic domain patterns. $\mathrm{SiO}_{2} / \mathrm{V} / \mathrm{CMS} / \mathrm{MgO} / \mathrm{CFS}$ and $\mathrm{SiO}_{2} / \mathrm{V} / \mathrm{CFS} / \mathrm{MgO} / \mathrm{CMS}$ trilayer structures exhibit a strikingly different magnetic behavior, which is due to pinhole coupling through the $\mathrm{MgO}$ barrier and a strong thickness dependence of the magnetic ordering in $\mathrm{Co}_{2} \mathrm{MnSi}_{\text {. }}$
\end{abstract}

Key words: Heusler, Photoemission Electron Microscopy, Micromagnetism PACS: 68.37.Xy, 75.50.Ee, 75.70.Kw

\section{Introduction}

Magnetic tunnel junctions (MTJ), i.e. two magnetic electrodes separated by a very thin tunneling barrier, are currently receiving great interest due to their technical importance in spintronics [1]. The applications range from hard disk read heads to memory cells in magnetic random access memories or magnetologic circuits. The tunneling magnetoresistance (TMR) effect in the diffusive limit can be quantitatively described by Jullière's model [2] as

$$
\mathrm{TMR}=\frac{R_{\uparrow \downarrow}-R_{\uparrow \uparrow}}{R_{\uparrow \uparrow}}=\frac{2 P_{1} P_{2}}{1-P_{1} P_{2}}
$$

with the spin polarization values at the Fermi level of the two electrodes $P_{1}$ and $P_{2}$ and the resistances $R_{\uparrow \downarrow}$ and $R_{\uparrow \uparrow}$ of the antiparallel and the parallel magnetization configuration, respectively. In this simplified model half-metals with a spin polarization of

Email address: a.kaiser@fz-juelich.de (A. Kaiser).
$100 \%$ at the Fermi level are expected to show an infinitely high TMR effect. By the use of half-metallic $\mathrm{La}_{2 / 3} \mathrm{Sr}_{1 / 3} \mathrm{MnO}_{3}$ electrodes a TMR effect of $1800 \%$ was already demonstrated at low temperatures 3 . At room temperature this effect vanishes due to the low Curie temperature of $\mathrm{La}_{2 / 3} \mathrm{Sr}_{1 / 3} \mathrm{MnO}_{3}$. Another promising class of half-metallic materials with noticeably higher Curie temperatures is the group of full-Heusler compounds 44. Several Cobalt-based Heusler alloys with Curie temperatures well above room temperature have been predicted to show $100 \%$ spin polarization at the Fermi level [5] and are therefore promising candidates for MTJ electrode materials. Dual-Heusler MTJs have already been realized with $\mathrm{Co}_{2} \mathrm{MnSi}$ electrodes and an $\mathrm{AlO}_{x}$ tunneling barrier reaching high TMR values [6]. By the use of epitaxially grown electrode-barrier combinations even higher values can be expected due to the reduction of defects and the onset of resonant tunneling mechanisms [7. Magnesium oxide $(\mathrm{MgO})$ 
provides compatible lattice constants to the above mentioned Heusler alloys allowing epitaxial growth of the MTJ structures [8].

The full-Heusler compounds of the type $\mathrm{X}_{2} \mathrm{YZ}$ are among the materials predicted to exhibit halfmetallic behavior with $100 \%$ spin polarization in the bulk [5]. $\mathrm{Co}_{2} \mathrm{MnSi}(\mathrm{CMS})$ and $\mathrm{Co}_{2} \mathrm{FeSi}$ (CFS) are two protagonists of this class, having similar lattice constants and providing structural compatibility to $\mathrm{MgO}$. Both materials have very high Curie temperatures of $985 \mathrm{~K}$ (CMS, 9]) and $1100 \mathrm{~K}$ (CFS, [10]), thereby fulfilling a prerequisite for their potential use in applications. The magnetic moment per formula unit is $5.07 \mu_{B}$ (CMS) and $6 \mu_{B}$ (CFS), respectively. The experimental results described below show that both materials have clearly distinguishable coercive fields. This opens a pathway to combine CFS and CMS in one MTJ in the sense of a pseudo spin-valve structure, i.e., without using exchange bias materials for the reference magnetization direction.

Up to now many macroscopic studies of the magnetic, electronic and structural properties of magnetic tunnel junctions have been carried out. From a fundamental point of view, but also with respect to technical aspects, the micromagnetic behavior of small elements cannot be neglected. The equilibrium magnetization of magnetic thin film elements is characterized by the minimum of the magnetic free energy, which is composed of several competing contributions, such as the exchange energy penalizing deviations from a homogeneous magnetization direction, the anisotropy energy describing the preferred orientation of the magnetization with respect to certain crystalline axes of the material, the Zeeman energy describing the interaction with the external field and the stray field energy connected with the demagnetizing field generated by the element itself [11. The stray field $\mathbf{H}_{\mathbf{d}}$ depends strongly on the dimensions and the shape of the magnetic element. Thus, by microstructuring the samples using lithographic techniques the influence of $\mathbf{H}_{\mathbf{d}}$ on different element sizes can be studied and compared to the effect of the other competing energy terms provided that these remain unchanged. In thin film layer stacks, an additional energy term comes in due to coupling mechanisms between the ferromagnetic layers, for instance, interlayer exchange coupling or Néel coupling. For the performance of MTJs, these coupling contributions may be very important and may have a considerable influence on the magnetic microstructure.
There is a variety of well-established techniques known for the investigation of the magnetic microstructure, such as magnetooptical Kerr microscopy, Lorentz microscopy, magnetic force microscopy, or scanning electron microscopy with spin-polarization analysis 12. Only few of them, however, combine magnetic sensitivity with chemical selectivity. The chemical selectivity is an indispensible precondition for the separation of the magnetic contributions in a layered system. One of these techniques is Photoemission Electron Microscopy (PEEM) in combination with polarized soft $\mathrm{x}$-ray synchrotron radiation. It is a powerful tool to element-selectively investigate the local domain structure with both high spatial [1314] and temporal [15 resolution, and has therefore been used as the main method in our studies.

\section{Experimental details}

Single films and trilayer structures have been prepared by magnetron sputtering onto Silicon substrates with a $20 \mathrm{~nm}$ Vanadium seed layer at room temperature, followed by an in-situ annealing step at $450^{\circ} \mathrm{C}$ (single films) and $300^{\circ} \mathrm{C}$ (trilayers with $\mathrm{MgO})$, respectively. During the sputtering process a weak in-plane magnetic field of $7 \mathrm{mT}$ was present in the deposition chamber. Afterwards the samples have not been exposed to any other magnetic field and have been studied in the as-grown state. The films have been capped with a thin Vanadium layer to prevent oxidation of the layer stack. X-Ray diffraction patterns showed the films to grow in a highly textured B2-phase. This is expected to result in a reduction of the spin-polarization and therefore a loss of the half-metallicity as compared to the preferred $\mathrm{L} 2_{1}$-phase [16]. The films have been subsequently microstructured by optical lithography and Argon ion beam milling into squares with areas ranging from $2 \times 2$ to $100 \times 100 \mu \mathrm{m}^{2}$. Prior to the microscopy experiments $M(H)$-curves have been measured using vibrating sample magnetometry in order to determine the coercive fields and magnetic moments. By the use of synchrotron radiation the static magnetic microstructure of the samples was investigated element-selectively with an Elmitec PEEM III at the variable polarization undulator beamline UE56/1-SGM at BESSY-II (Berlin). For this purpose the photon energy was tuned to the corresponding $\mathrm{L}_{3}$-absorption edge of $\mathrm{Mn}, \mathrm{Fe}$, or Co and the XMCD asymmetry value 


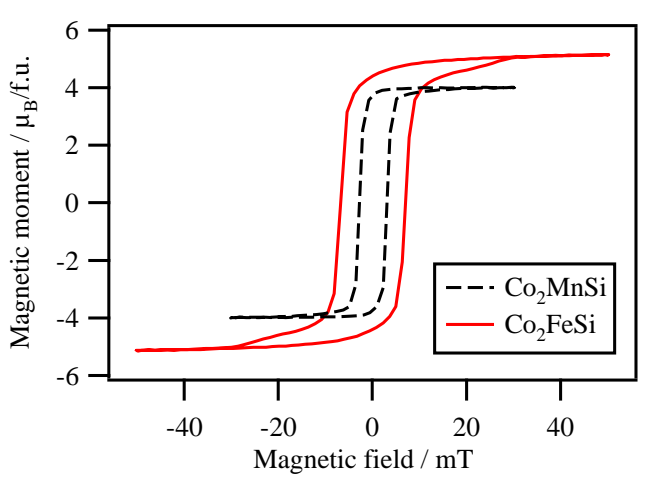

Fig. 1. In-plane $M(H)$-curves of $20 \mathrm{~nm} \mathrm{Co}_{2} \mathrm{MnSi}(\mathrm{CMS})$ and $\mathrm{Co}_{2} \mathrm{FeSi}$ (CFS) thin films measured with vibrating sample magnetometry, showing the difference in saturation magnetization and coercive fields.

$A=\left(I_{\sigma+}-I_{\sigma-}\right) /\left(I_{\sigma+}+I_{\sigma-}\right)$ was calculated for each pixel with $I_{\sigma \pm}$ being the intensity values for right and left circularly polarized light.

\section{Results and discussion}

\subsection{Single films}

Fig. 1 shows the $M(H)$-curves of $20 \mathrm{~nm}$ thick single films of CMS and CFS measured with the magnetic field applied in the film plane. They exhibit clearly distinguishable coercive fields of $2.8 \mathrm{mT}$ (CMS) and $6.5 \mathrm{mT}(\mathrm{CFS})$ and magnetic moments per formula unit of $4.0 \mu_{B}$ (CMS) and $5.2 \mu_{B}$ (CFS). The reduction of the magnetic moments compared to the bulk values can be explained by atomic site disorder and interface effects. The hysteresis measurements have been carried out with the magnetic field applied along different in-plane angles in order to determine a possible global magnetic anisotropy in the samples. For both samples no sizable in-plane anisotropy could be found. This in-plane isotropy in the samples was also confirmed by FMR measurements [17.

The magnetic structure of both materials (Fig. 2) is strongly influenced by the polycrystalline nature of the films. In both films multidomain states are formed with the local magnetization direction laterally varying due to anisotropy fluctuations. However, a perpendicular magnetization direction is strongly suppressed by the demagnetizing field, which forces the average magnetization direction into the film plane. A direct comparison of the images in Figs. 2(a) and 2(b) reveals clear differences between CMS and CFS. Both films develop

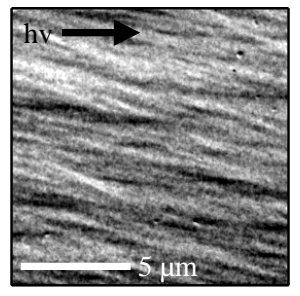

(a) CMS

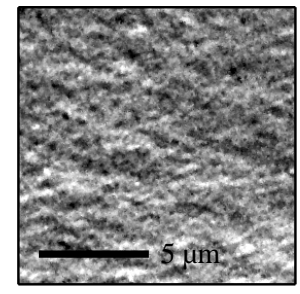

(b) CFS
Fig. 2. Magnetic domain patterns of extended (a) CMS and (b) CFS films. The magnetic contrast originates from XMCD at the $\mathrm{Co}_{3}$ edge. In all images the direction of light incidence is from left to the right.

a magnetization ripple with the average magnetization vector pointing perpendicular to the domain walls [18]. The existence of magnetization ripple can be attributed to the polycrystalline nature of the films leading to a strong variation of the local magnetocrystalline anisotropy associated with each grain. The average wavelength of the periodic ripple structure can be correlated with the average crystallite size [19]. In our measurements the CMS film showed a more textured magnetization distribution with a preferred axis, giving rise to the "feathery" pattern. By contrast, the CFS films reveal a graintype domain structure, which is possibly related to a smaller grain structure and higher disorder in the film. The average ripple wavelength in CMS is about $1.89 \mu \mathrm{m}$ and significantly larger than the value for the respective CFS films $(0.68 \mu \mathrm{m})$.

In comparing with Fig. 1 it is also interesting to note that the shorter ripple wavelength in CFS is in fact related to a higher coercivity. This finding is consistent with the picture that the smaller grains and higher disorder in the film block the magnetization reversal process, for example, via domain wall pinning.

In the next step, we structured the films into small micrometer-sized elements. In the magnetic domain patterns, the difference between the two materials becomes even more obvious: Under the influence of the shape-induced demagnetizing field, the response of elements of comparable size is distinctly different. The CMS film develops a so-called concertina or buckling pattern (shown in fig. 3(c) also known from Permalloy elements in a similar thickness range [20]. It is formed by alternating low-angle walls with the local magnetization direction varying around the average magnetization. With decreasing element size the effect of the demagnetizing field be- 


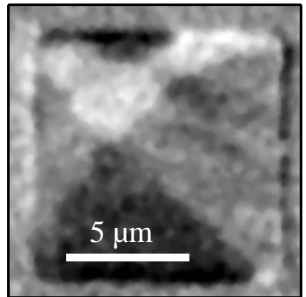

(a)

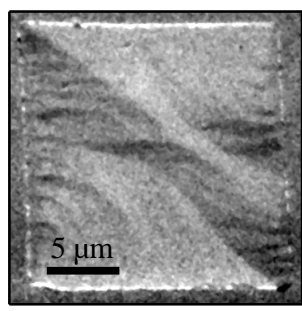

(c)

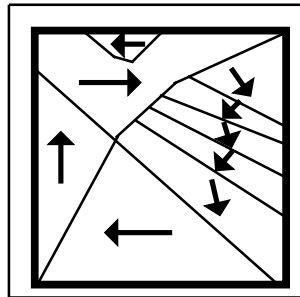

(b)

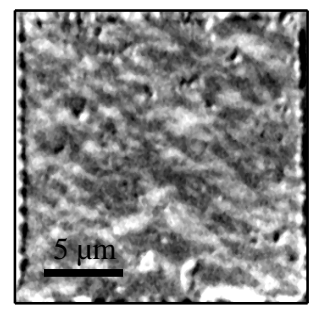

(d)
Fig. 3. Magnetic domain structures in patterned CMS and CFS elements. (a) CMS 10x10 $\mu \mathrm{m}^{2}$, (b) Sketch of domain pattern in CMS $10 \times 10 \mu \mathrm{m}^{2}$, (c) CMS $20 \times 20 \mu \mathrm{m}^{2}$, (d) CFS $20 \times 20 \mu \mathrm{m}^{2}$. In all cases the magnetic contrast has been obtained at the Co $\mathrm{L}_{3}$ edge.

comes stronger and successively simpler flux-closure patterns reminiscent of Landau states start to form (Fig. 3(a) and 3(b), which are still accompanied by buckling structures. The latter disappear for elements in the micrometer regime. However, the occurrence of the buckling state is not necessarily the magnetic ground state configuration, but may arise due to a local energetic minimum caused by neighboring domains blocking each other.

A completely different response is observed in the CFS films. Even under the influence of the demagnetizing field in small $10 \times 10 \mu \mathrm{m}^{2}$ elements (Fig. $3(\mathrm{~d})$ the polycrystalline nature of the film is dominating the magnetization pattern and the fine-grained domain structure remains essentially unchanged from that observed in the extended film (Fig. 2(b) . This result shows that the intrinsic anisotropy of the CFS-film is much stronger than the demagnetizing field of the square element.

\subsection{Trilayer: $\mathrm{Co}_{2} \mathrm{MnSi}(20 \mathrm{~nm}) / \mathrm{MgO}$ (3 nm) $/ \mathrm{Co}_{2} \mathrm{FeSi}$ (2 $\mathrm{nm}$ )}

In a second step the single Heusler films have been combined into trilayer structures with a $\mathrm{MgO}$ interlayer of $3 \mathrm{~nm}$ thickness. In order to separate the magnetic response of the individual layers in this stack,

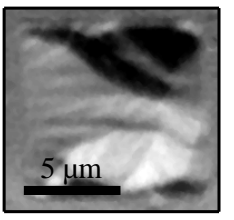

(a) $\mathrm{Co}-\mathrm{L}_{3}$

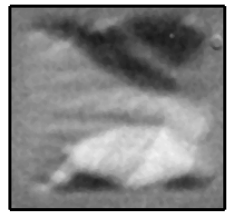

(b) $\mathrm{Fe}-\mathrm{L}_{3}$

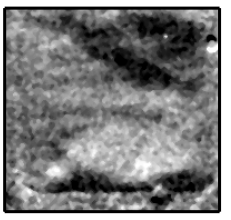

(c) $\mathrm{Mn}-\mathrm{L}_{3}$
Fig. 4. Element-selective domain imaging in the layer system $\mathrm{CMS} / \mathrm{MgO} / \mathrm{CFS}$, revealing a parallel magnetic coupling of the CFS and CMS films. In the first two images ( $\mathrm{Co}, \mathrm{Fe})$ the same scaling of the magnetic contrast is used, while in the third image $(\mathrm{Mn})$ the measured contrast is five times smaller and has been upscaled for better visibility. The element size is $10 \times 10 \mu \mathrm{m}^{2}$.

the full versatility of soft $\mathrm{x}$-ray PEEM is needed. By tuning the photon energy of the incident beam to the $\mathrm{L}_{3}$-absorption edges of the specific elements (Co, Mn, Fe) the magnetization of both ferromagnetic layers can be investigated independently. At the Co edge, the response of both the CFS and CMS layers is probed, whereas XMCD at the Fe and Mn edges provides the magnetic information of the CFS and CMS layer, respectively.

In the case of the CMS $(20 \mathrm{~nm}) / \mathrm{MgO}(3 \mathrm{~nm}) /-$ CFS (2 nm) structure (our nomenclature always starts with the bottom layer), the resulting domain images for a $10-\mu \mathrm{m}$ square element are compiled in Fig. 4. The thickness of the CFS layer has been chosen as $2 \mathrm{~nm}$, in order to be able to probe the bottom CMS layer. It has to be noted that due to the lower film thickness of the top layer, a direct comparison to the results of the single film measurements in chapter 3.1 is not possible, since the film thickness may strongly influence the domain configuration. As we will show below, however, magnetic coupling phenomena are the dominant effect determining the top layer magnetization distribution.

The information depth for the total yield detection mode in PEEM is limited to a few nanometers in metals 21. As a consequence, the Mn-signal is rather weak and the magnetic contrast is only about $20 \%$ of that of the Co and Fe signals. In order to facilitate the comparison of the images in Fig. 4 the Mn-contrast has thus been scaled up to the same level as for Co and Fe.

A direct comparison of the three domain patterns reveals identical structures for the Co and Fe data. Although the contrast on the Mn is weaker and the image has a lesser signal-to-noise figure, the main domain features, but also some of the concertina structures can be clearly discerned. This indicates 


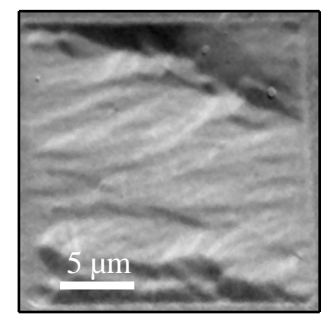

(a)

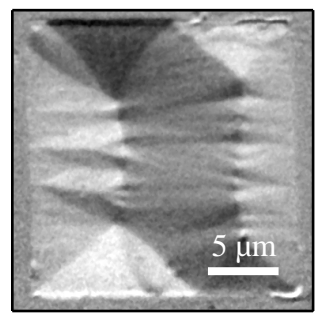

(b)
Fig. 5. Comparison of magnetic domain patterns acquired at the $\mathrm{Co}_{3}$ edge of $20 \times 20 \mu \mathrm{m}^{2}$ square elements of both trilayers $\operatorname{CMS}(20 \mathrm{~nm}) / \mathrm{MgO}(3 \mathrm{~nm}) / \mathrm{CFS}(2 \mathrm{~nm})(\mathrm{a})$ and $\mathrm{CFS}(20 \mathrm{~nm}) / \mathrm{MgO}(3 \mathrm{~nm}) / \mathrm{CMS}(2 \mathrm{~nm})(\mathrm{b})$.

that the two Heusler films are indeed not magnetically independent, but exhibit a considerable ferromagnetic coupling through the $\mathrm{MgO}$ interlayer. The reasons for this coupling can be a roughness-induced Néel/orange-peel mechanism 22] or pinholes in the $\mathrm{MgO}$ layers, which favor a ferromagnetic contact between the CFS and CMS layer through a direct exchange interaction.

The data also show that the micromagnetic behavior of the $\mathrm{CMS}(20 \mathrm{~nm}) / \mathrm{MgO}(3 \mathrm{~nm}) / \mathrm{CFS}(2 \mathrm{~nm})$ trilayer stack is strongly dominated by the thicker CMS film. The domain patterns shown in Fig. 4 and in 5(a) resemble the situation of the single CMS film. While for the larger $20 \times 20 \mu^{2}$ element the magnetic microstructure is determined by the polycrystalline structure of the film and a magnetization ripple is formed, for $10 \times 10 \mu \mathrm{m}^{2}$ elements the competition between anisotropy-induced ripple formation and flux closure domains due to the demagnetizing field created a very different equilibrium configuration.

\subsection{Trilayer: $\mathrm{Co}_{2} \mathrm{FeSi}(20 \mathrm{~nm}) / \mathrm{MgO}(3 \mathrm{~nm}) /$ $\mathrm{Co}_{2} \mathrm{MnSi}(2 \mathrm{~nm})$}

As a surprise the magnetic microstructure changes drastically, when the layer sequence of the trilayer is reversed, i.e., the bottom layer is formed by a $20 \mathrm{~nm}$ thick CFS film. Instead of the anisotropydominated multi-domain ripple structure observed in Fig. 3(d) we find a completely different behavior. The average domain size increased and a lowremanence magnetization pattern consisting of two antiparallel Landau domains are formed as shown in Fig. 5(b) Some of the $90^{\circ}$-walls have been replaced by an additional domain with two low-angle walls.
This configuration has been referred to as "tulip" state 23 . The $180^{\circ}$-walls between neighboring antiparallel domains are modified by a high density of cross-ties. By the formation of cross-ties the magnetostatic energy is reduced, since $180^{\circ}$-walls are replaced by energetically more favorable $90^{\circ}$-walls 24].

The element-selective analysis of the domain structure reveals similar magnetic structures at the Co- $\mathrm{L}_{3}$ and $\mathrm{Fe}-\mathrm{L}_{3}$ edges, which is consistent with domain structure resulting from the CFS film. On the other hand, we do not find a magnetic contrast at the $\mathrm{Mn}^{-\mathrm{L}_{3}}$ edge. As our method has been shown to be able to even pick up the Mn XMCD from the buried layer in the $\mathrm{CMS} / \mathrm{MgO} / \mathrm{CFS}$ sandwich, we must conclude that the CMS film in our trilayer is nonmagnetic - at least at room temperature, where the experiments have been carried out. This behavior may be attributed to a strong thickness dependence of the CMS magnetic moment in thin films below $8 \mathrm{~nm}$ thickness supporting the results of previous XAS and FMR measurements [25|26|27]. The strongly reduced Curie temperature in the $2 \mathrm{~nm}$ CMS film may be explained by interdiffusion at the interface leading to a higher atomic disorder. Furthermore, this result seems to indicate that the $\mathrm{MgO}$ barrier in this layer has only a negligible density of pinholes, because a direct exchange coupling to the bottom CFS layer should also result in a common Curie temperature for both layers.

Room-temperature $M(H)$-measurements of the $\mathrm{CMS} / \mathrm{MgO} / \mathrm{CFS}$ sandwich revealed a soft magnetic behavior with a coercive field of $2.5 \mathrm{mT}$ and a total magnetic moment of $4.6 \mu_{B} / f . u$.. With the $2 \mathrm{~nm}$ thin CMS layer being magnetically inactive the difference in the magnetic properties of this trilayer and the single CFS film can only be explained by the different annealing temperatures of both samples. By the lower annealing temperature the crystalline structure is not fully established leading to a weaker magnetocrystalline anisotropy. Similar results have indeed been reported by Oogane et al [28]. Thus, the relative influence of the demagnetizing field may be stronger. As a consequence, the formation of Landau-type flux-closure patterns is favored over a multi-domain magnetization ripple.

\subsection{Effect of annealing on magnetic microstructure}

In order to gain more insight into the role of the annealing process and temperature, we have also 


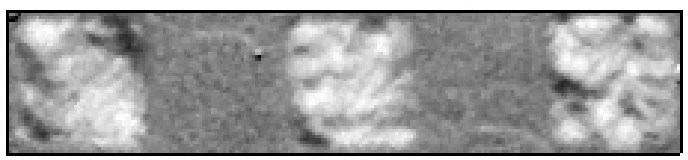

(a) $200^{\circ} \mathrm{C}$

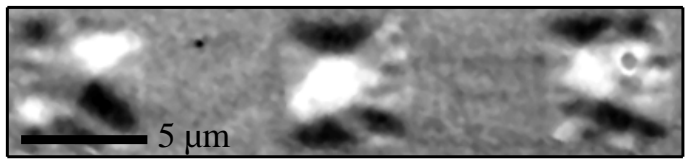

(b) $270^{\circ} \mathrm{C}$

Fig. 6. Snapshots of the magnetization distribution in a $\mathrm{CMS} / \mathrm{MgO} / \mathrm{CFS}$ sandwich during annealing to different temperatures taken at the Cobalt $\mathrm{L}_{3}$ absorption edges. Size of the square elements: $5 \times 5 \mu \mathrm{m}^{2}$. Both images refer to the same grey scale.

performed first in-situ annealing experiments on the above samples in the photoemission microscope, using a built-in heating stage. It is a well-known fact that the deposition or annealing temperature of Heusler alloys has a strong impact on their structural, magnetic and electric properties 29. In particular, the annealing should lead to a reduction of the site disorder. Therefore, the Heusler phase formation will also have a strong effect on the micromagnetic structure of the individual films and of the layer stack. As an example, we have chosen a $\mathrm{CMS}(20 \mathrm{~nm}) / \mathrm{MgO}(3 \mathrm{~nm}) / \mathrm{CFS}(2 \mathrm{~nm})$ trilayer in the as-grown state for the annealing experiments. As a new approach to the problem, we have taken the magnetic domain images directly at different sample temperatures and not at room temperature after the annealing. In this way, we are able to directly follow the evolution of magnetic changes in the system. Note that this approach requires the Curie temperature to be sufficiently high to yield a magnetic signal in PEEM.

The as-grown layer stack exhibits only a quite weak magnetic dichroism at the $\mathrm{Co}_{2} \mathrm{~L}_{3}$-edge. As a consequence, the domain pattern observed at room temperature is rather diffuse and noisy. This result does not change very much up to a temperature of about $200^{\circ} \mathrm{C}$ (Fig. 6(a)). Between $230^{\circ} \mathrm{C}$ (not shown) and $270^{\circ} \mathrm{C}$, however, we observe a sudden change of the domain pattern (Fig. 6(b) : Despite the elevated temperature, the contrast becomes stronger and the domain pattern more regular. This change can be linked to a (partial) formation of the Heusler phase. In order to prove this link, we have also acquired additional spectroscopic information by taking local X-Ray absorption spectra across the

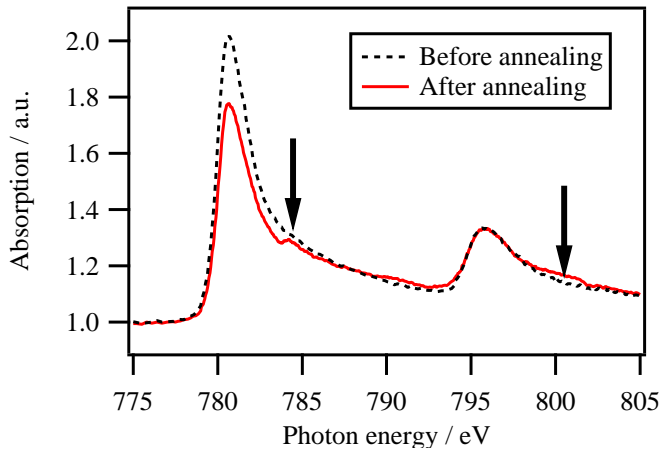

Fig. 7. X-ray absorption spectra of the $\mathrm{Co}_{2,3}$ edges before and after the annealing step. Features changing during the annealing procedure are marked by the arrows.

Co- $\mathrm{L}_{2,3}$-edges (Fig. 7). Comparing the spectra in the as-grown with those of the annealed state (after the annealing step), we can clearly see that the change in the magnetic microstructure is accompanied by the appearance of an additional spectral feature on the high-energy side of the main absorption lines. This additional feature has previously been identified as an indication for increased atomic and magnetic order in these materials 25] and is therefore sometimes referred to as "Heusler peak". Our experimental approach provides a direct access to the formation dynamics of the Heusler phase and will be expanded to other systems in the future.

\section{Conclusions}

Our element-selective domain imaging experiments reveal the complexity of the magnetic microstructure in Heusler-based thin film systems. The results also show that the micromagnetic structure depends on fine details of the formation process of the Heusler phases. The ferromagnetic coupling observed in the dual-Heusler trilayers is due to pinhole formation and roughness-induced Néel coupling. It must be overcome by an improvement of the preparation conditions. The surprising difference of the magnetic behavior between the $\mathrm{CMS} / \mathrm{MgO} / \mathrm{CFS}$ and $\mathrm{CFS} / \mathrm{MgO} / \mathrm{CMS}$ trilayer structures is due to a strong thickness dependence of the magnetic ordering in CMS and must be taken into account for the construction of magnetic tunneling junctions.

Acknowledgement 
We gratefully acknowledge the financial support by the DFG within the SFB 491.

\section{References}

[1] S. Wolf, D. Awschalom, R. Buhrman, J. Daughton, S. von Molnar, M. Roukes, A. Chtchelkanova, D. Treger, Science 294 (2001) 1488.

[2] M. Jullière, Phys. Lett. A 54 (1975) 225.

[3] M. Bowen, M. Bibes, A. Barthélémy, J. Contour, A. Anane, Y. Lemaître, A. Fert, Appl. Phys. Lett. 82 (2003) 233.

[4] F. Heusler, Verh. Dtsch. Phys. Ges. 12 (1903) 219.

[5] R. de Groot, F. Mueller, P. Engen, K. Buschow, Phys. Rev. Lett. 50 (1983) 2024.

[6] Y. Sakuraba, M. Hattori, M. Oogane, Y. Ando, H. Kato, A. Sakuma, T. Miyazaki, H. Kubota, Appl. Phys. Lett. 88 (2006) 192508.

[7] S. Yuasa, T. Nagahama, A. Fukushima, Y. Suzuki, K. Ando, Nature Materials 3 (2004) 868.

[8] M. Yamamoto, T. Marukame, T. Ishikawa, K. Matsuda, T. Uemura, M. Arita, J. Phys. D: Appl. Phys. 39 (2006) 824.

[9] P. Webster, K. Ziebeck, Landolt-Börnstein New Series III/9c (1988) 75 .

[10] S. Wurmehl, G. Fecher, H. Kandpal, V. Ksenofontov, C. Felser, H. Lin, J. Morais, Phys. Rev. B 72 (2005) 184434.

[11] A. Hubert, R. Schäfer, Magnetic Domains, Springer, 1998.

[12] H. Hopster, H.P. Oepen (eds.), Magnetic Microscopy of Nanostructures, Springer, 2005.

[13] G. Schönhense, J. Phys.: Cond. Matt. 11 (1999) 9517.

[14] J. Stöhr, S. Anders, IBM J. Res. Develop. 44 (2000) 535.

[15] G. Schönhense, H. Elmers, S. Nepijko, C.M. Schneider, Adv. Imag. Electron Phys. 142 (2006) 159.

[16] S. Picozzi, A. Continenza, A. Freeman, Phys. Rev. B 69 (2004) 94423.

[17] K. Zakeri, unpublished result (2007).

[18] H. Hoffmann, IEEE Trans. Magn. 4 (1968) 32.

[19] A. Baltz, W. Doyle, J. Appl. Phys. 35 (1964) 1814

[20] D. Chumakov, J. McCord, R. Schäfer, L. Schultz, H. Vinzelberg, R. Kaltofen, I. Mönch, Phys. Rev. B 71 (2005) 014410.

[21] B. Frazer, B. Gilbert, B. Sonderegger, G. De Stasio, Surf. Sc. 537 (2003) 161.

[22] L. Néel, C. R. Acad. Sci. 255 (1962) 1676.

[23] A. Hubert, M. Rührig, J. Appl. Phys. 69 (1991) 6072.

[24] E. Huber Jr, D. Smith, J. Goodenough, J. Appl. Phys. 29 (1958) 294.

[25] J. Schmalhorst, S. Kämmerer, M. Sacher, G. Reiss, A. Hütten, A. Scholl, Phys. Rev. B 70 (2004) 24426.

[26] W. Wang, M. Przybylski, W. Kuch, L. Chelaru, J. Wang, Y. Lu, J. Barthel, H. Meyerheim, J. Kirschner, Phys. Rev. B 71 (2005) 144416.

[27] B. Rameev, F. Yildiz, S. Kazan, B. Aktas, A. Gupta, L. Tagirov, D. Rata, D. Bürgler, P. Grünberg, C.M. Schneider, S. Kämmerer, G. Reiss, A. Hütten, phys. stat. sol. (a) 1 (2006) 10.
[28] M. Oogane, R. Yilgin, M. Shinano, S. Yakata, Y. Sakuraba, Y. Ando, T. Miyazaki, J. Appl. Phys. 101 (2007) 09J501.

[29] S. Kämmerer, The Heusler alloy $\mathrm{Co}_{2} \mathrm{MnSi}$ in thin films, Ph.D. thesis, Univ. Bielefeld (2003). 